\title{
Comparative Examination of the Life Quality in Hemodialysis Patients and Kidney Transplant Recipients in the Educational-Medical Centers of Ahvaz
}

\author{
Shahla Assadi Hovyzian, ${ }^{1}$ Sadigheh Fayazi, ${ }^{2,}{ }^{*}$ Asaad Sharhani, ${ }^{3}$ Mohammad Ayoubi, ${ }^{4}$ and Sajade \\ Mosaviasl $1^{5}$ \\ ${ }^{1}$ MSc, Golestan Hospital Clinical Research Development Unit, Ahvaz Jundishapur University of Medical Sciences, Ahvaz, Iran \\ ${ }^{2}$ MSc, Nursing Care Research Center in Chronic Diseases, Department of Nursing, Nursing and Midwifery School, Ahvaz Jundishapur University of Medical Science, Ahvaz, \\ Iran \\ ${ }^{3}$ Department of Epidemiology, School of Public Health, Shahid Beheshti University of Medical Sciences, Tehran, Iran \\ ${ }^{4}$ MSc in Cellular- Molecular Biology, Microbiology Branch, Science Faculty, Ahvaz Shahid Chamran University, Ahvaz, Iran \\ ${ }^{5} \mathrm{MSc}$, Student Research Committee, Ahvaz Jundishapur University of Medical Sciences, Ahvaz, Iran \\ "Corresponding author: Sadigheh Fayazi, Nursing Care Research Center in Chronic Diseases, Department of Nursing, Nursing and Midwifery School, Ahvaz Jundishapur \\ University of Medical Science, Ahvaz, Iran. Tel: +98-9161136441, E-mail: sadighe_fa@yahoo.com
}

Received 2016 August 15; Revised 2016 December 28; Accepted 2017 January 10.

\begin{abstract}
Background: Kidney chronic failure and its replaced remedies make the patient exposed to a wide range of physical, mental, economic, and social problems and as the life quality is affected by them, the life quality would be changed. Therefore, evaluation of the life quality by specific evaluation tools and based on demographic information help the patients' problems to be dealt with principally. In this study, we examine the life quality of hemodialysis patients and kidney transplant recipients in Ahvaz.

Methods: This study is a cross sectional study to compare the life quality of patients who had kidney transplantation or underwent hemodialysis treatment. Sampling was conducted based on purposeful method and the life quality of 70 patients and 70 kidney transplant recipients referring to medical center and the people who had the criterion for entering the study were examined by a questionnaire of kidney diseases' life quality $\left(\mathrm{KDQOL}_{-} \mathrm{SF}_{36}\right)$; then, after collecting data, they were compared by applying statistical tests including T-test and Chi-square test.

Results: The mean total scores of the life quality did not show a statistically significant difference in two groups $(\mathrm{P}=0.344)$, but the kidney transplantation group with a significant mean difference obtained better scores in the dimensions of general health (P $=0.002)$, physical health $(\mathrm{P}=0.000)$, sleep $(\mathrm{P}=0.028)$, limitation in playing a role $(\mathrm{P}=0.000)$, and physical limitation $(0.002)$ in comparison with hemodialysis group. The two groups did not show any significant difference in dimensions of disorder in social natural function and social relations, physical pain, limitation because of pain, vivacity, coping with disease, inner emotion related to disease, self-knowledge, emotional issues, and sexual activities.

Conclusions: Although kidney transplantation could increase the patients' life quality in some dimensions, in the kidney transplantation group the people still encounter their previous problems and are in need of receiving more care to keep their transplant kidney. Based on results, therefore, educational - medical centers must predict and implement necessary arrangements to increase the life quality in both groups.
\end{abstract}

Keywords: Life Quality, Patients, Kidney Transplantation, Hemodialysis

\section{Background}

Chronic kidney failure is considered as one of the main problems of general health (1). The mean prevalence of chronic kidney failure in Europe and U.S.A and England is 171,366 , and 100 cases in one million per year, respectively (2). Based on the report of the Management Center for Transplantation and Special Diseases, Ministry of Health, the population of patients suffering from Kidney failure in our country, is 320,000 cases of which $49 \%, 48 \%$, and $1 \%$ use the treatment methods: transplantation, hemodialysis, and peritoneal hemodialysis, respectively. The increasing procedure of these patients in the world indicates that the number of patients who undergo replacement treatments including kidney transplantation, peritoneal hemodialysis, and hemodialysis is increasing, so as a result of the side effects of this disease and its treatments, all aspects of a usual life for this group of patients are disrupted (1).

Treatment method of dialysis makes many limitations for a patient, so that hemodialysis patients have a different life in comparison with others. They make themselves emotion-dependent and do not enjoy freedom to program 
their personal life. They feel insecure about their future life because many changes have been made in their life means, family state, and position. The prevalent stressful factors in this disease consist of a feeling of powerlessness, lack of controlling disease and treatment; administering imposed treatment; having limitation as a result of medical diet; change in body image of themselves; economic problems; and ability to hold down a job and sexual affairs (3, 4).

Moreover, transplantation is considered as a trustworthy and important medical option in replaced remedies of kidney and, based on different sources, is followed by increasing the life quality of patients and allowing the client to have a more natural life (5-7); but, as this transplantation operation suppresses immune system, it is followed by important side effects and medical care is needed $(8,9)$. Today, the main reason for disability and death is transplantation, and cardiac-vascular disease has the second place (10). Another problem is the possibility of malignancy; the patients who received immune-suppressing treatment for the long run are exposed to cancer more than others $(5,8$, 9). Moreover, these patients suffer from the many kinds of stress because of infection danger, fear of being hospitalized continually, change in personality, limitation in physical movement because of the hurting transplant kidney, change in body appearance and putting on weight which affects their life mentally, economically, and socially $(6,10$, 11).

Therefore, considering partly double prevalence of the two treatments and the side effects followed by each of them, several studies have been conducted on comparing two treatments in all of which the effect of treatments on the life quality has been studied to compare these treatments (7-12).

Life quality is known as a multidimensional concept including physical aspects, symptoms of disease, and the effect of disease and treatment in life and mental, social, family, and economic states which are affected by personal experience and the perception of one from life and would be changed by passing time $(10,11,13)$. Evaluating the life quality as an outcome of health level in a hygiene science dimension has a wide range of applications and patients' life quality as one of the evaluation indexes of treatment results has drawn many specialists' attention $(9,12)$; not only is it the aim of treating the patients suffering from kidney failure to save their life, but also it is to improve their life quality (13); as far as, the assessment of the life quality help patients' problems to be paid attention to principally and medical methods are revised and also the patients suffering from chronic disease are affected by medical and care policy more than others; therefore, the discussion of the life quality in these patients has drawn the peo- ple's and chairmen's serious attention $(14,15)$. Moreover, researchers evaluated the life quality of patients suffering from chronic problems through different methods in order to help medical and care programming.

Namadi, in his study, applied the specific tool of the life quality of patients suffering from kidney failure (KDQOL$\mathrm{SF}$ ) to compare the life quality of hemodialysis and transplantation groups. Nemadi indicated that the life quality of transplant patients was better than that of hemodialysis patients' (13). In spite of increasing the life quality in transplant patients, results of some studies have shown that the state of general health in kidney recipients in some dimensions of the life quality is poorer than dialysis or there was no significant difference (16-18). In Tayyebi's study on comparing the life quality of both groups using a life quality questionnaire of $\mathrm{SF}_{36}$, the results indicated that transplant patients were better than dialysis patients just in two dimensions of general health and physical function and both groups were similar in other dimensions of the life quality $(14,17)$.

In his study on the examination the life quality of hemodialysis and kidney transplant patients, Abbas Zadeh concluded that there was no statistical significant difference in the life quality of both groups in three dimensions of physical function, physical pain, and social function. In hemodialysis patients, the lowest score was related to freshness dimension and the highest one to physical function dimension; and in kidney transplant patients, the lowest score was related to general health dimension and the highest one to the limitation in role dimension because of physical problems (12).

Therefore, the need for a more accurate examination of these patients' problems by applying specific evaluation tools would be perceptible while most of domestic studies, except for Namadi (13), applied general tools for examining the life quality $\left(\mathrm{SF}_{36}\right)$. Moreover, so far, no accurate examination has been done to compare these two groups of patients in Ahvaz city which has a specific climate condition in term of bad weather and its side effects for kidney transplant and hemodialysis patients are more than other parts of the country $(12,19)$. These problems made the researcher examine and compare the life quality of dialysis patients with transplant patients applying more specific and accurate tools for kidney patients' life quality (KDQOL-SF) in Ahvaz.

\section{Methods}

This is an applied cross sectional descriptive-analytical study to compare the life quality of patients who had kidney transplant or underwent hemodialysis treatment in Ahvaz in 2011. The sample sizes, by using the formula, were 
calculated 21 in each group; to increase the accuracy rate, 70 patients were selected in each group. Patients were selected on a consecutive sequential basis until the completion of the sample volume. A total of 70 hemodialysis patients and 70 kidney transplant recipients who had the criteria entered the study. The inclusion criteria were: age range of 17 to 70 years, being at least six months after their hemodialysis starting date or kidney transplantation, and being a citizen of Ahvaz. Samples were selected from research environment including kidney transplantation ward, nephrology ward of governmental specialty, and subspecialty hospitals: Imam Khomeini and Golestan within four months. Ethical code of study was taken from the deputy for research, Ahvaz Jundishapur University of Medical Sciences (Code of ethical approval: 92s75). Exclusion criteria were being at less than six months after their treatment, patients whose transplantation had been rejected for any reason, the patients whose place of living was not Ahvaz, and those who were not satisfied with the participation in the study. After explaining the aim of the study and assuring the participants that their information would not be revealed, they were asked conscious satisfaction; then, demographic data including clinical-personal characteristics such as age, gender, marital status, and level of education, job, amount of income, duration of disease and treatment, the amount of hemoglobin, creatine, weight and mental disorder were collected by applying a questionnaire of demographic information. In order to examine the life quality of both groups, they were provided with standardized questionnaire of kidney patients' life quality (KDQOL -SF ${ }^{\mathrm{TM}} 1.3$ ). This questionnaire has been designed by adaptation of tools such as KDQOl Version, ESRD, and WHOQOL modified in accordance with Asian culture; it is quite specific and short, does not make patients tired and is easy to understand. The Iranian version of this questionnaire has been translated to Persian by Yekaninejad et al. and it enjoys good psychometric characteristics, so that after evaluating Cronbach's coefficient, the range of stability has been evaluated form $77 \%$ for physical dimension to $92 \%$ for symptom dimension and 79 to $92 \%$ for questionnaire validity. Other studies show that the questionnaire of KDQOL- SF has high stability and enjoys high reliability among ESRD patients (20). Other studies show that the questionnaire of KDQOL- SF has high stability and enjoys high reliability among ESRD patients (18).

This questionnaire contains dimensions of general health (three questions); limitation on daily activities (10 questions); limitation on the role because of physical problems (four questions); limitation on the role because of emotional problems (three questions); disorder in social natural function (one question); physical pain and limitation because of pain (two questions); vivacity (nine ques- tions), amount of disorder in social relation (one question); self-knowledge (four questions); coping with disease (four questions); emotional issues (six questions); physical burden of disease (12 questions); disorder in daily life (eight questions); sexual activities (three questions); sleep state (one question); problems of sleep and awakeness (three questions); satisfaction with family and friends (two questions); Job and income (one question), and effect of disease on job and income (one question). In order to score the questionnaire of the life quality, the raw number of each group would be separately evaluated. Therefore, scores of questions specific to each dimension would be added up and the result number would be divided by the number of that dimension's questions and at the end, the score of that dimension would be achieved with a high score indicating the favorite quality of life.

After collecting data from each under-research unit, it was analyzed by applying statistical software of SPSS ${ }_{18}$, independent T-test for the quantitative variables, and Chi-square test for dual-mode variable. Moreover, we compared common dimensions between two groups and scores of two groups' life quality were generally compared.

\section{Results}

In this study, life quality of 70 kidney failure patients who underwent hemodialysis treatment and 70 kidney failure patients who underwent kidney transplantation and were inhabitants of Ahvaz were examined by applying the KDQOL- SF questionnaire; then, data were compared by applying statistical tools of independent t-test, and Chi-square test; the results are observable in two tables: 1) demographic characteristics (Table 1) and 2) comparing scores of the two groups' life quality in each dimension (Table 2). As Table 1 shows, $37.1 \%$ of dialysis patients were women and $61.4 \%$ of them were men and $60 \%$ of patients in the transplantation group were men and $40 \%$ of them were women, which indicates the higher proportion of men in two groups. Most of dialysis patients were married but the percentage of married (55.7\%) and single (44.3\%) people in the transplantation group was partly the same. A total of $50 \%$ of the hemodialysis group mentioned nephropathy as a reason for affliction while most of the transplantation group (42.9\%) selected option of 'others' as a reason for transplantation. About $54.3 \%$ of dialysis patients mentioned that they started their treatment from 1 to 3 years ago, but $77.1 \%$ of transplant patients did not mention the date of starting their treatment. A total of $2.9 \%$ of hemodialysis patients and $18.5 \%$ of transplant patients had mental problems and this difference was significant between two groups $(\mathrm{p}=0.030)$. Other demographic characteristics are shown in Table 1. 
Table 1. Demographic Characteristics of Under-Research Units

\begin{tabular}{|c|c|c|c|c|c|c|}
\hline \multirow{2}{*}{\multicolumn{2}{|c|}{ Personal Characteristics }} & \multicolumn{2}{|c|}{ Hemodialysis } & \multicolumn{2}{|c|}{ Transplant } & \multirow[t]{2}{*}{ PValue } \\
\hline & & Frequency & Percentage & Frequency & Percentage & \\
\hline Age & & & & & & 0.000 \\
\hline & $>30$ & 4 & 5.7 & 10 & 14.7 & \\
\hline & $40-30$ & 6 & 8.6 & 24 & 35.3 & \\
\hline & $50-40$ & 18 & 25.7 & 16 & 23.5 & \\
\hline & $<50$ & 42 & 60 & 20 & 28.5 & \\
\hline Gender & & & & & & 0.581 \\
\hline & Man & 43 & 61.4 & 42 & 60 & \\
\hline & Woman & 26 & 37.1 & 28 & 40 & \\
\hline Educatio & & & & & & 0.000 \\
\hline & Illiterate & 21 & 30 & 3 & 4.3 & \\
\hline & Primary school & 21 & 30 & 9 & 12.9 & \\
\hline & Secondary school & 10 & 14.3 & 17 & 24.3 & \\
\hline & High school & 15 & 21.4 & 29 & 41.4 & \\
\hline & University & 3 & 4.3 & 12 & 17.1 & \\
\hline Marital s & status & & & & & 0.000 \\
\hline & Single & 6 & 8.6 & 31 & 44.3 & \\
\hline & Married & 64 & 91.4 & 39 & 55.7 & \\
\hline Reason $\mathrm{fc}$ & for kidney failure & & & & & 0.000 \\
\hline & Hypertension & 11 & 15.9 & 14 & 21.2 & \\
\hline & Glomerulonephritis & 4 & 5.8 & 11 & 16.7 & \\
\hline & Nephropathy & 35 & 50.7 & 11 & 16.7 & \\
\hline & Others & 19 & 27.5 & 30 & 45.5 & \\
\hline Starting & treatment & & & & & 0.000 \\
\hline & $3-1$ & 38 & 54.3 & 5 & 31.3 & \\
\hline & $6-3$ & 10 & 4.3 & 5 & 31.3 & \\
\hline & $>3$ & 2 & 2.9 & 3 & 37.5 & \\
\hline Job & & & & & & 0.061 \\
\hline & Unemployed & 52 & 37.4 & 59 & 37.4 & \\
\hline & Employed & 18 & 25.7 & 11 & 15.7 & \\
\hline Hemoglo & obin & & & & & 0.002 \\
\hline$>8$ & & 15 & 21.7 & 12 & 25 & \\
\hline & $10-8$ & 31 & 44.9 & 33 & 68.8 & \\
\hline & $12-10$ & 23 & 33.3 & 3 & 6.3 & \\
\hline Amount & of urea & & & & & 0.009 \\
\hline & $>20$ & 4 & 5.8 & 7 & 14 & \\
\hline & $30-20$ & 13 & 18.8 & 19 & 38 & \\
\hline & $<30$ & 52 & 75.4 & 24 & 48 & \\
\hline Creatine & & & & & & 0.000 \\
\hline & $5.1-5.0$ & 4 & 0.58 & 26 & 40.6 & \\
\hline & $>5.1$ & 65 & 94.2 & 38 & 59.4 & \\
\hline Weight & & & & & & 0.090 \\
\hline & $50-30$ & 8 & 0.114 & 11 & 20.4 & \\
\hline & $70-50$ & 36 & 51.4 & 32 & 59.3 & \\
\hline & $>70$ & 26 & 37.1 & 11 & 20.4 & \\
\hline Spirit & & & & & & 0.030 \\
\hline & Without problem & 2 & 2.9 & 10 & 18.5 & \\
\hline & With problem & 68 & 97.1 & 44 & 81.5 & \\
\hline
\end{tabular}

Table 2 indicates the score of both groups' life quality in a different dimension and the comparison of it be- tween the two groups. Based on the results of question number 1 , transplantation group with a mean of 2.813 have 
Table 2. Comparing the Mean Score of Life Quality in Each Dimension in the Two Groups: Kidney Transplantation and Dialysis

\begin{tabular}{|c|c|c|c|c|c|}
\hline \multirow[t]{2}{*}{ Questions } & \multicolumn{2}{|c|}{ Kind of Treatment } & \multirow[t]{2}{*}{ Mean Difference } & \multirow[t]{2}{*}{ P Value } & \multirow[t]{2}{*}{ Confidence Interval } \\
\hline & Hemodialysis & Kidney transplantation & & & \\
\hline 1. General health & $2.18 \pm 0.11$ & $2.81 \pm 0.16$ & $0.62 \pm 0.19$ & 0.002 & $1.01-0.24$ \\
\hline 2. Health in comparison with last year & $2.54 \pm 0.14$ & $4.72 \pm 0.34$ & $2.17 \pm 0.37$ & 0.000 & $2.91-1.44$ \\
\hline 3. Limitation on daily activities & $1.98 \pm 0.08$ & $2.56 \pm 0.08$ & $0.57 \pm 0.11$ & 0.000 & $0.81-0.33$ \\
\hline 4. Imitation on role because of physical problems & $0.34 \pm 0.04$ & $1.09 \pm 0.10$ & $0.75 \pm 0.11$ & 0.000 & $0.97-0.52$ \\
\hline 5. Limitation on role because of emotional problems & $0.34 \pm 0.04$ & $0.77 \pm 0.15$ & $0.46 \pm 0.13$ & 0.001 & $0.73-0.19$ \\
\hline 6. Disorder in social natural function & $3.09 \pm 0.18$ & $3.13 \pm 0.15$ & $0.04 \pm 0.23$ & 0.862 & $0.51-0.42$ \\
\hline 7. Physical pain & $3.10 \pm 0.17$ & $2.74 \pm 0.19$ & $0.36 \pm 0.25$ & 0.167 & $0.15-0.87$ \\
\hline 8. Limtation because of pain & $2.62 \pm 0.15$ & $2.91 \pm 0.15$ & $0.29 \pm 0.22$ & 0.188 & $0.73-0.14$ \\
\hline 9. Vivacity & $3.26 \pm 0.11$ & $3.04 \pm 0.10$ & $0.21 \pm 0.15$ & 0.159 & $0.08-0.52$ \\
\hline 10. Amount of disorder in social relation & $3.294 \pm 0.2$ & $3.367 \pm 0.17$ & $0.073 \pm 0.26$ & 0.785 & $0.60-0.45$ \\
\hline 11. Knowing him/herself & $2.835 \pm 0.07$ & $3.007 \pm 0.09$ & $0.171 \pm 0.11$ & 0.144 & $0.40-0.05$ \\
\hline 12. Coping with disease & $2.301 \pm 0.12$ & $2.610 \pm 0.16$ & $0.308 \pm 0.2$ & 0.138 & $0.71-0.10$ \\
\hline 13. Emotional issues & $3.948 \pm 0.11$ & $4.010 \pm 0.09$ & $0.061 \pm 0.14$ & 0.676 & $0.35-0.22$ \\
\hline 14. Physical burden of disease & $3.209 \pm 0.11$ & $3.617 \pm 0.1$ & $0.408 \pm 0.15$ & 0.011 & $0.72-0.09$ \\
\hline 15. Disorder in daily life & $2.719 \pm 0.12$ & $3.245 \pm 0.11$ & $0.525 \pm 0.17$ & 0.002 & $0.86-0.18$ \\
\hline 16. Sexual activities & $2.675 \pm 0.26$ & $2.650 \pm 0.21$ & $0.025 \pm 0.33$ & 0.941 & $0.64-0.69$ \\
\hline 17. Sleep state & $5.812 \pm 0.31$ & $6.822 \pm 0.33$ & $0.010 \pm 0.45$ & 0.028 & $1.91-0.10$ \\
\hline 18. Problems of sleep and wakeness & $3.647 \pm 0.11$ & $4.102 \pm 0.14$ & $0.455 \pm 0.18$ & 0.014 & $0.81-0.09$ \\
\hline 19. Satisfaction with family and friends & $3.398 \pm 0.07$ & $3.117 \pm 0.1$ & $0.280 \pm 0.12$ & 0.030 & $0.02-0.53$ \\
\hline 20. Job and income & $0.085 \pm 0.03$ & $0.242 \pm 0.05$ & $0.157 \pm 0.0 .06$ & 0.012 & $0.27-0.03$ \\
\hline 21. Effect of the disease on job and income & $0.471 \pm 0.06$ & $0.300 \pm 0.05$ & $0.171 \pm 0.08$ & 0.037 & $0.01-0.33$ \\
\hline 22. Giving a score to your health & $5.685 \pm 0.31$ & $7.028 \pm 0.23$ & $1.342 \pm 0.39$ & 0.001 & $2.12-0.56$ \\
\hline
\end{tabular}

Table 3. Comparing the Mean. Total Score of Life Quality in Two Groups of Kidney Transplantation and Dialysis

\begin{tabular}{lccccc}
\hline Total Score of Health and Well-Being & hemodialysis & Kidney Transplantation & Mean Difference & P Value & Confidence Interval \\
\hline 1. Total score of life quality & $61.727 \pm 2.99$ & $64.872 \pm 1.53$ & $3.144 \pm 3.29$ & 0.344 \\
\hline
\end{tabular}

assessed their health better than the hemodialysis group with a mean of $2.185(\mathrm{P}=0.002)$. Moreover, in comparison with the last year, the transplantation group with a mean difference of 2.178 have assessed their health better than hemodialysis group, which is a statistically significant difference $(\mathrm{P}=0.000)$.

In terms of limitation on daily activities and playing a role because of physical health state and emotional issues which consist of questions number 3 to 5 , the score of the transplantation group in each question was higher than the score of dialysis group and it showed a significant difference. As a result, the hemodialysis patients have more limitation on their activities and playing a role.
The two groups did not show any significant difference in the dimensions of disorder in social function, physical pain, cognitive issues, coping with disease, and vivacity. Based on the results of questions 14 and 15, hemodialysis patients, with a significant difference, suffer from physical problems more than the transplantation group because of side effects of kidney failure and its effect on kidney function $(\mathrm{P}=0.002$ and $\mathrm{P}=0.011)$ and frequently a high level of waste substance in these patients' blood and great dependency on the hemodialysis apparatus have been mentioned as its reasons.

Moreover, results of questions 17 and 18 indicate that the sleep state of the transplantation group was signifi- 
cantly better than dialysis $(\mathrm{P}=0.014$ and $\mathrm{P}=0.028)$, which can be related to decreasing dialysis' side effects such as skin itching because of modification of the amount of urea and creatine and, as a result, patient' comfort during night sleep. Based on the results of question number 19, satisfaction with family and friends in the dialysis group has been more than the transplantation group, which is statistically significant $(\mathrm{P}=0.030)$ and can be justified by the married status of most of dialysis people and also by the experience of a feeling of becoming a burden by hemodialysis because of senility and frequently referring to hospital and the necessity of having an attendant.

Based on the results of question number 20, kidney transplantation group, with a significant mean difference, was employed more than the dialysis group while this group considered their health state as an obstacle to have high- income jobs more than dialysis group ( $\mathrm{P}=0.037)$; however, this is justified by the mean age of transplantation group which was mostly in the range of 30 to 40 years old while most of the dialysis were older than 50 years and were mostly retiree.

In question number 22 related to giving a score to their health, kidney transplantation group gave a higher score to their health in a comparison with the dialysis group and this is statistically significant $(P=0.001)$.

Regarding the results of the questions of the KDQOLSF questionnaire, the mean score of life quality in the dialysis group (61.727) and the kidney transplantation group (64.872) was achieved and there was no statistical significant difference in the total score of the life quality of both groups (Table 2 ).

\section{Discussion}

The present study was conducted with the aim of comparing the life quality of hemodialysis and transplant patients in Ahvaz educational- medical centers. Regarding the results of the research, life quality in the transplantation group, with a significant mean difference, was better than the dialysis group in some dimensions (Table 2) and in some cases, improving the score of life quality of the kidney transplantation group in these dimensions was related to demographic characteristics of the subjects. According to Table 1, significant differences in some demographic characteristics between the two groups was observed, For example, $60 \%$ of the subjects in the hemodialysis group were in the range of over 50 years old and $35.3 \%$ of the subjects in the transplantation group were in the age range of 30 to 40 years old, which was a significant age difference ( $\mathrm{P}-0.000)$, because of the high risk transplant in older age which tends to be lower in patients with advanced age for the transplant. An evaluation of the rela- tionship between age and total score of the life quality by applying ANOVA test indicated a significant relationship between age and the life quality in the kidney transplantation group (0.038), which is consistent with the study conducted by Mollahadi and Vosughi $(8,19)$. Moreover, there were significant differences between the scores of the life quality in general health and physical function dimensions with age group in the study conducted by Raaeisifar, and in the above-mentioned dimensions, the health state was better in the age group under 25 years old, which is consistent with our study and is inconsistent with the results of the study conducted by Zsofia Kovacs (16).

Moreover, a significant relation was observed between the scores of life quality with education in both groups so that the highest percentage of education in both groups was high school degree, but university degree in the transplantation group (17.1\%) was more than the dialysis group (4.3\%), which is a statistically significant difference ( $\mathrm{P}=$ $0.000)$. The education difference between the two groups could be due to the lower age in transplant groups and it is more likely for them to have studied. Therefore, there were statistical significant differences between the level of education and the total score of the life quality in both groups $(\mathrm{P}=0.001)$. In the study by Vosughi, comparing the difference of the life quality in terms of education levels became significant in the dialysis patients and became more desirable by increasing levels of education, which is consistent with our study (8).

There was the largest percentage of dialysis (74.3) and transplant (84.3) among the unemployed and a significant relationship was observed between the total score of the life quality and job in both groups by applying ANOVA test $(\mathrm{P}$ for dialysis $=0.000$ and for kidney transplantation group $=0.004$ ). Moreover, $77.4 \%$ of hemodialysis patients and $70 \%$ of transplant patients were unemployed and in hemodialysis patients, mean of the life quality in unemployed persons was significantly lower than employed and retired persons $(\mathrm{P}=0.012)$.

A significant relationship was achieved between weight and the total sore of life quality in the hemodialysis group $(\mathrm{P}=0.003)$. Moreover, the amount of urea and creatine (94.2\% and $75.4 \%$, respectively) in hemodialysis patients was not normal, which indicated the relationship of urea with the life quality in hemodialysis group approaching significance $(\mathrm{P}=0.064)$. Moreover, physical effect of disease and its side effect of sleep disorder and emotional issues in transplantation group were lower than hemodialysis group which conformed to results of studies by Amirkhani, Tayyebi $(1,14,17)$. The reason for that can be justified in the way that high amount of urea and creatine of hemodialysis group's blood make night itching and muscular pain and as a result causes 
sleep disorder followed by drowsiness during the day and emotional problems. The results of other study showed that sleep disorder and depression in transplant patients is lower than hemodialysis patients (18).

The psychological problems that transplant patients were allocated to in Table 1 show a significant difference between the two groups $(\mathrm{P}=0.030)$. That is because recipients had experienced clinically significant levels of anxiety and experienced high levels of negative effects of immunosuppressant medication (21).

Therefore, modifying factors such as weight, education, employment, blood factors, and effective relationship between members of the family can increase the life quality of both groups; therefore, the chairmen of healthmedical services must design and regulate supportive centers for this vulnerable group of society.

In comparison with hemodialysis group, one of the other reasons for improving the score of life quality in the transplantation group in these dimensions (health in comparison with last year, the limitation because of physical and emotional problems, sleep state and the score given to their health) is the positive effect of kidney transplantation on patients' perception of health and freedom feeling followed by increased self-respect. Virzi considers dropping hemodialysis as a factor to improve the life quality in kidney transplantation patients (22); therefore, the lesser limitation of transplantation group in activities and playing role can be justified regarding dropping dependency to the hemodialysis apparatus, eliminating the diet and activity limitation and actively returning to the society (2325 ). In other studies, hemodialysis patients received lower scores in the dimensions of physical health, which is consistent with the results of our study (16). Studies conducted by Vosughi, Shakeri, Amirkhani, and Abbas Zadeh also approved this subject $(1,8,12,26)$.

The results of this study showed that, however, the score of life quality in the kidney transplantation group was higher than dialysis group, this difference was not statistically significant $(\mathrm{P}=0.344)$; because there were no significant differences in dimensions of disorder in social natural function and social relations, physical pain, limitation in role because of pain, vivacity, coping with disease, inner emotion related to disease, self-knowledge, emotional issues, and sexual activities of both groups; and even life quality in the hemodialysis group, with a significant difference, was better than the transplant patients (Table 2) in the dimensions of satisfaction with family and friends (0.030) and the effect of the disease state on job and income $(\mathrm{P}=0.37)$. In the study conducted by Tayyebi, satisfaction with family was at an average level in both groups (14).

Many studies consider the reason for the same pain in both group's existence of severe muscular and bone pain in patients who take cycloseporin $(24,26-29)$. Based on the results of Abbas Zadeh' study, there were no significant differences in three dimensions of physical function, physical pain, and social function in both groups (12). Based on Tayyebi' study, there was no significant difference in the dimensions of physical function, motion limitation, pain, energy, social function, limitation on playing an emotional role, and mental health in both groups which is consistent with our study (14).

Although kidney transplantation can increase life quality in the patients in some dimensions $(26,28)$, physical and mental effect such as stress resulting from rejection of transplantation, change in mind picture from body because of immunosuppressive medicine and danger of making infection can affect social function and decrease life quality of these patients; then, regarding huge economic, social, and spiritual-mental expense of kidney transplantation operation, the need for more studies and finding possible reasons for decreasing the life quality level in the patients is felt. The weak points of the study were that the patients who had undergone kidney transplantation had previously undergone dialysis, which may impact their quality of life. Alternatively, as this study was conducted in Ahvaz, more study must be conducted for examining the effect of existing conditions in this city in terms of health, education, and climate on life quality in the transplant patients.

\subsection{Conclusion}

Based on the results of this study, patients suffering from kidney chronic failure must be supported by multilateral health-medical systems; and if necessary, hemodialysis patients must be put on a fast list of kidney transplantation. However, multilateral support of patients before and after operating transplantation must be paid attention to by designing and making multi-specialization systems based on cooperative function; as kidney transplant operation itself affects the life quality of the patients due to the huge expense of operation, and stress resulting from the rejection of transplantation, taking immunosuppressive medicine and its physical and mental side effect. Therefore, it is necessary to regulate a model of care in kidney transplant patients extracted from their needs in the kidney transplantation process based on the chronic care model and self-management model.

\section{Acknowledgments}

This article is part of a student research project of M.Sc. in nursing confirmed by deputy research of Ahvaz 
Jundishapur University of Medical Sciences (Code of ethical approval: 92s75) and is conducted by the financial support of this university; hereby, we would like to express our gratitude and appreciation to this deputy and also our special thanks go to the members of Golestan Hospital Clinical Research Development Unit and all patients who participated in the present study.

\section{References}

1. Amirkhani M, Nouhi E, Jamshidi $H$. The comparative survey of life quality in renal transplant recipients, peritoneal dialysis and hemodialysis patients in kerman in the year 2013 [In Persian]. J Fasa Univ Med Sci. 2014;4(1):126-33.

2. Hadian B, Anbari K, Heidari R. Epidemiologic study of end stage renal disease and related risk factors in patients under hemodialysis in Lorestan province [In Persian]. Yafteh. 2015;16(3):44-53.

3. Baghaei Kake M, Rahimi S, Adib M, Kazem Nejad Leili E, Monfared A. Predictive personal factors of quality of life in hemodialysis Patients [In Persian]. Holist Nurs Midwifery. 2015;24(4):9-19.

4. Omrani HR, Raeisi D, Seyyedzadeh A. Quality of life of hemodialysis patients and the factors that affect in Kermanshah (1389) [In Persian]. Mon J Med Sci Kermanshah. 2013;16(8).

5. Raaeisifar A, Tayyebi A, Ebadi A. An investigation of quality of life in kidney transplant patients [In Persian]. Iran J Crit Care Nurs. 2011;4(3):149-52.

6. Fayazi S, Asadizaker M, Shahrouz A. Comparison of quality of life between haemodialysis and renal transplant patients [In Persian]. World Crit Care Nurs. 2008;6(4):69-72.

7. Anwar W, Ezzat H, Mohab A. Comparative study of impact of hemodialysis and renal transplantation on cognitive functions in ESRD patients. Nefrologia. 2015;35(6):567-71. doi: 10.1016/j.nefro.2015.07.004. [PubMed: 26441130].

8. Vosughi M, Movahedpoor A. Comparison of quality of life in hemodialysis and renal transplant in hospital of Ardabil centers [In Persian]. JArdabil Univ Med Sci. 2009;9(2):171-2.

9. Zeraaty A. Compare the quality of life in patients undergoing hemodialysis and peritoneal dialysis centers Hospital of Imam Reza (AS) and Ghaem Mashhad city [In Persian]. Med J Mashhad. 2010;53(3):169-75.

10. Namdar A, Bigizade S, Nagafipour S. Health- related quality of life in dialysis patients [In Persian]. JJahrom unit Med Sci. 2013;10(4):19-27.

11. Rambod M, Shabani M, Shokrpour N, Rafii F, Mohammadalliha J. Quality of life of hemodialysis and renal transplantation patients. Health Care Manag (Frederick). 2011;30(1):23-8. doi: 10.1097/HCM.ob013e3182078ab6. [PubMed: 21248544].

12. Abbas Zadeh A, Jvanbkhtyan R, Salehi S, Motevaselian M. Comparison of quality of life in hemodialysis patients and recipients KidneyTransplantation [In Persian].J Shaheed Sadoughi Univ Med Sci. 2011;18(5):4618 .

13. Namadi M, Movahedpur A. Comparson of quality of life in hemodialysis patients and renal transplant in centers hospitals of Ardabil [In Persian]. JArdabil Univ. 2009;9(2):171-9.

14. Tayyebi A, Salimi S, Mahmoudi H, Tadrisi S. Review the renal transplantation patients' quality of life by using kidney transplantation questionnaire (KTQ-25) [In Persian]. Iran J Crit Care Nurs. 2010;3(3):125-8.
15. Tol A, Esmaeil Shahmirzadi S, Moradian Sorkhkolaee M,Azam K. Determination of quality of life of dialysis and kidney transplant patients compared to healthy people. J Res Health Systems. 2012;7(6):1170-7.

16. Kovacs AZ, Molnar MZ, Szeifert L, Ambrus C, Molnar-Varga M, Szentkiralyi A, et al. Sleep disorders, depressive symptoms and health-related quality of life-a cross-sectional comparison between kidney transplant recipients and waitlisted patients on maintenance dialysis Nephrol Dial Transplant. 2011;26(3):1058-65. doi: 10.1093/ndt/gfq476. [PubMed: 20685829].

17. Molnar-Varga M, Molnar MZ, Szeifert L, Kovacs AZ, Kelemen A, Becze A, et al. Health-related quality of life and clinical outcomes in kidney transplant recipients. Am J Kidney Dis. 2011;58(3):444-52. doi: 10.1053/j.ajkd.2011.03.028. [PubMed: 21658828].

18. Yekaninejad MS, Mohammadi Zeidi I, Akaberi A, Golshan A, Pakpour A. Validity and reliability of the kidney disease quality of life - short form (KDQOL-SF ${ }^{\mathrm{TM}} 1.3$ ) in Iranian patients [In Persian].J North Khorasan Univ Med Sci. 2012;4(2):273.

19. Mollahadi M, Tayyebi A, Ebadi A, Daneshmandi M. Comparison between anxiety,depression and stress in hemodialysis and kidney transplantation patients [In Persian].J Crit Care. 2010;2(4):153-6.

20. Abedi Samakoosh M, Aghaie N, Gholami F, Shirzad M, Yosefi E, Teymoorzadeh Baboli M. Assesment dialysis adequacy in hemodialysis patients of Qaemshar Razi hospital in 2012 [In Persian].J Mazandaran Univ Med Sci. 2012;23(107).

21. Zimmermann T, Pabst S, Bertram A, Schiffer M, de Zwaan M. Differences in emotional responses in living and deceased donor kidney transplant patients. Clin Kidney J. 2016;9(3):503-9. doi: 10.1093/ckj/sfw012. [PubMed: 27274840].

22. Virzi A, Signorelli MS, Veroux M, Giammarresi G, Maugeri S, Nicoletti A, et al. Depression and quality of life in living related renal transplantation. Transplant Proc. 2007;39(6):1791-3. doi: 10.1016/j.transproceed.2007.05.011. [PubMed: 17692614].

23. Anees M, Hameed F, Mumtaz A, Ibrahim M, Saeed Khan MN. Dialysisrelated factors affecting quality of life in patients on hemodialysis. Iran J Kidney Dis. 2011;5(1):9-14. [PubMed: 21189427].

24. Mini AM, Emmanuel J, Unni VN, Deepa AR, Shameena A. Evaluation of quality of life in hemodialysis and renal transplant patients. Int J Pharm Health Sci. 2010;1:77-83.

25. Sayin A, Mutluay R, Sindel S. Quality of life in hemodialysis, peritoneal dialysis, and transplantation patients. Transplant Proc. 2007;39(10):3047-53. doi: 10.1016/j.transproceed.2007.09.030. [PubMed: 18089319].

26. Shakeri A, Abbaszadeh MR, Valizadeh H. Determination of quality of life of dialysis and kidney transplant patients compared to healthy people [In Persian]. Kerman University; 2014.

27. Emami Naeini A. Thyroid function markers in patients receiving hemodialysis and peritoneal dialysis for chronic kidney disease [In Persian].J Isfahan Med School. 2013;31(224).

28. Yango AF, Gohh RY, Monaco AP, Reinert SE, Gautam A, Dworkin LD, et al. Excess risk of renal allograft loss and early mortality among elderly recipients is associated with poor exercise capacity. Clin Nephrol. 2006;65(6):401-7. doi: 10.5414/CNP65401. [PubMed:16792134].

29. Chavalitdhamrong D, Gill J, Takemoto S, Madhira BR, Cho YW, Shah $\mathrm{T}$, et al. Patient and graft outcomes from deceased kidney donors age 70 years and older: an analysis of the Organ Procurement Transplant Network/United Network of Organ Sharing database. Transplantation. 2008;85(11):1573-9. doi: 10.1097/TP.0b013e31817059a1. [PubMed: 18551062]. 